\title{
Characterization of small leucine-rich proteoglycans in aortic valves of patients with aortic valve stenosis
}

\author{
Hiroshi Furukawa $^{* 1,2}$, Masahide Chikada ${ }^{1}$, Michiyo K. Yokoyama ${ }^{2}$, Mitsumi Arito ${ }^{2}$, Manae S. Kurokawa ${ }^{3}$, Toshiyuki Sato ${ }^{2}$, Masaaki Sato ${ }^{2}$, \\ Kazuki Omoteyama ${ }^{2}$, Naoya Suematsu ${ }^{2}$, Toshiya Kobayashi ${ }^{1}$, Masahiro Sagane ${ }^{1}$, Hirotoshi Suzuki ${ }^{1}$, Takashi Ando ${ }^{1}$, Tomohiro Kato ${ }^{2}$, and \\ Takeshi Miyairi ${ }^{1}$ \\ ${ }^{1}$ Department of Cardiovascular Surgery, St. Marianna University School of Medicine, Japan \\ ${ }^{2}$ Clinical Proteomics and Molecular Medicine, St. Marianna University Graduate School of Medicine, Japan \\ ${ }^{3}$ Disease Biomarker Analysis and Molecular Regulation, St. Marianna University Graduate School of Medicine, Japan
}

\begin{abstract}
We previously reported aberrant glycosylation of lumican, one of small leucine-rich proteoglycans (SLRPs), in aortic valves in patients with aortic valve stenosis (AS). Extending the study, we here characterized other SLRPs of proline/arginine-rich end leucine-rich repeat protein (PRELP), decorin, and biglycan in AS valves. Specifically, thickened and calcified (AS-C) areas and apparently non-thickened and non-calcified (AS-N) areas of aortic valves were sampled in 8 patients with AS. Then amounts of three molecules with or without removal of N-glycosylation by PGNase were compared between the AS-C and AS-N areas. As a result, the amounts of PRELP with moderate and/or low amounts of $\mathrm{N}$-glycosylation were found decreased in the AS-C areas compared to the AS-N areas. The amounts of decorin with both glycosaminoglycan (GAG) and N-linked oligosaccharides were decreased in the AS-C area compared to the AS-N area. Moreover, the amounts of biglycan with GAG and N-linked oligosaccharides were decreased, instead, the amounts of biglycan without GAG were increased in the AS-C areas compared to AS-N areas. These quantitative and qualitative changes of the three SLRPs detected here may be involved in the progression of AS changes of aortic valves.
\end{abstract}

\section{Introduction}

Aortic valve stenosis (AS), caused mainly by degenerative changes with calcification, is one of the most common cardiovascular diseases. The morbidity rate of AS increases with age. Macroscopically, thickening and calcification are observed in irregular areas of aortic valves. The changes gradually progress and lead to narrowing of the valve orifice, which brings about various symptoms such as chest pain and dyspnea [1]. It is well accepted that AS develops through three processes of lipid accumulation, inflammation, and calcification [2-5]. These processes transform valvular interstitial cells into osteoblastic ones, which results in calcification of valve tissue [6-7]. However, precise pathophysiology remains to be elucidated.

Aortic valves contain abundant extracellular matrix (ECM). The main components of the aortic valve ECM are collagens, elastin, and proteoglycans (PGs) [8]. PGs are characterized by a core protein to which sulphated glycosaminoglycan (GAG) and oligosaccharides are covalently attached as side chains [9]. PGs work as hydrators, stabilizers, and space fillers of ECM [9].

We previously reported that lumican, a PG which belongs to small leucine-rich proteoglycans (SLRPs), was insufficiently glycosylated in thickened and calcified areas compared to apparently non-thickened and non-calcified areas of identical AS valves [10]. SLRPs, a group of the leucine-rich repeat (LRR) superfamily, are characterized by a central LRR domain flanked on both $\mathrm{N}$ - and $\mathrm{C}$ - terminal sides by conserved cysteine motifs [11-13]. Until now more than 10 SLRPs has been reported. SLRPs are thought to form framework of collagenous tissue by interacting with fibrillar collagen [14] and by protecting collagen fibrils from proteolytic damage [11]. SLRPs have been also reported to be involved in regulation of tissue inflammation and calcification
[15-17]. Thereby, we there speculated that the aberrant glycosylation of lumican may be involved in the development of thickening and calcification of aortic valves in AS. Based on these backgrounds, we here investigated whether other SLRPs showed quantitative and/ or qualitative differences in thickened and calcified areas compared to apparently non-thickened and non-calcified areas of identical AS valves, focusing on three SLRPs of proline/arginine-rich end leucinerich repeat protein (PRELP), biglycan, and decorin.

\section{Methods}

\section{Patients and clinical samples}

Nine patients with AS were enrolled in this study. Aortic valve samples were obtained during therapeutic aortic valve replacement for AS. All the samples were obtained with written informed consent. This study was approved by the institutional ethics committee of St. Marianna University School of Medicine. One of the 9 AS valve leaflets was used for photographic presentation in Figure 1. The remaining 8 leaflets were subjected to analyses (Three males and five females, average 68.8 years old, range 43-80 years old ) Six out of the 8 samples were identical with those used in our previous study [10].

Correspondence to: Hiroshi Furukawa, MD, Department of Cardiovascular Surgery, St. Marianna University School of Medicine, 2-16-1 Sugao, Miyamae, Kawasaki, Kanagawa, 216-8511 Japan, Tel: +81-44-977-8111 (ext. 3230); FAX: +81-44-976-5792; E-mail: hirofuru0801-med@yahoo.co.jp

Received: October 15, 2016; Accepted: October 26, 2016; Published: October 28, 2016 


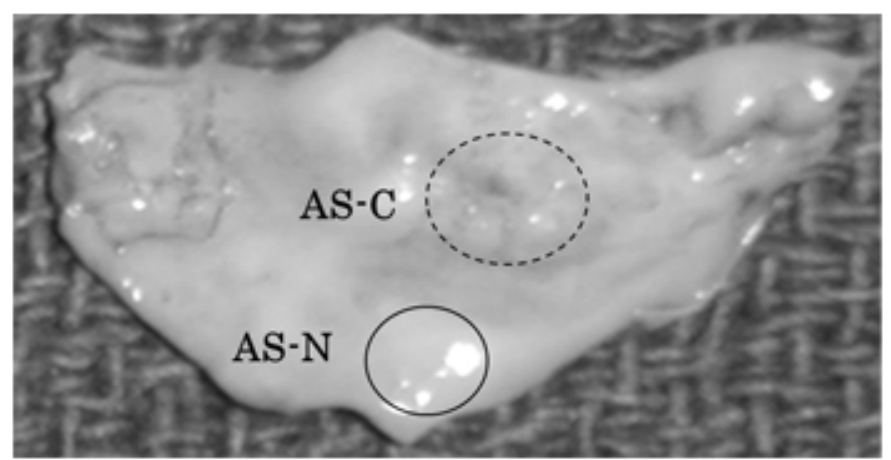

Figure 1. Preparation of aortic valve tissue samples from an AS-C area and an AS-N area of an identical valve.

\section{Protein preparation}

In each of the 8 AS aortic valve leaflets, thickened and calcified (ASC) areas and apparently non-thickened and non-calcified (AS-N) areas were separately sampled. The valve tissue samples were respectively subjected to protein extraction (Figure 1). Specifically, the samples were washed in phosphate-buffered saline to eliminate blood contaminants and then were minced and homogenized in a cell lysis buffer $(7 \mathrm{M}$ urea, $2 \mathrm{M}$ thiourea, 4\% 3-[(3-cholamidopropyl) dimethylammonio]-1propamesulfonate) using HG30 homogenizer (Hitachi Koki Co., Ltd., Tokyo). The samples were then frozen and thawed 5 times and centrifuged at $4^{\circ} \mathrm{C}$ for 30 minutes. The supernatants containing extracted proteins were collected and stored at $-80^{\circ} \mathrm{C}$ until use. A part of the extracted proteins were de-glycosylated by the treatment with Peptide:N-glycosidase F (PNGase F, Sigma, MO, USA) at the ratio of 50 units PNGase $\mathrm{F}$ per $\mathrm{mg}$ protein at $37^{\circ} \mathrm{C}$ for 3 hours and then were used in western blot.

\section{Gel electrophoresis and western blot (WB)}

Gel electrophoresis was performed as described previously [18]. Briefly, for one dimensional (1D) gel electrophoresis, protein samples were separated by $12.5 \%$ sodium dodecyl sulfate-polyacrylamide gel electrophoresis (SDS-PAGE) under reducing conditions. The separated proteins were transferred onto polyvinylidene difluoride (PVDF) membranes or nitrocellulose membranes. For two dimensional (2D) gel electrophoresis, the protein samples were subjected to isoelectric focusing (IEF) using gels with the range of $\mathrm{pH}$ 3.0-11.0. The IEFseparated proteins were then further separated by $12.5 \%$ SDS-PAGE. Proteins separated by 2DE were transferred onto PVDF membranes or nylon membranes. Then the membranes were subjected to WB. When needed, the membranes were stained with a fluorescent dye (SYPRO Ruby, Invitrogen Co., CA, USA) before WB.

WB was also performed as described previously [18]. Briefly, the membranes were reacted with antibodies to human PRELP (abcam, Cambridge, UK), Decorin (abcam), Biglycan (abcam) for 1 hour. The membranes were then washed and reacted with horseradish peroxidase (HRP)-labeled antibodies to rabbit IgG (Dako, Denmark) for 1 hour. After washing, bound antibodies were detected by 3 , 3'-diaminobenzidine, tetrahydrochloride (Dojin, Kumamoto, Japan) or by ECL select (GE Healthcare). Intensity of the detected bands in western blot was measured by an image analyzer.

\section{Statistical analysis}

Statistical significance was calculated using Student's $t$-test. A value of $p<0.05$ was considered to be statistically significant.

\section{Results}

We sampled tissue samples separately from a thickened and calcified (AS-C) area and an apparently non-thickened and noncalcified (AS-N) area of an identical aortic valve in each of the enrolled 9 patients with AS. A representative photograph of the aortic valves for sampling is shown in Figure 1. We then characterized PRELP, decorin, and biglycan from both the quantitative and qualitative aspects using 8 samples (one was used for the photograph presentation in Figure 1). As the qualitative analysis, we investigated glycosylation conditions of the molecules.

\section{Comparison of PRELP between the AS-C and AS-N areas in AS aortic valves}

We investigated expression of PRELP in the protein samples from the AS-C and AS-N areas by $1 \mathrm{D}-\mathrm{WB}$. As a result, PRELP was detected as a broad band with molecular weights (MWs) of approximately 60$70 \mathrm{kDa}$ in both areas in identical valves in 8 patients with AS (Figure 2 (a) left). A quantitative comparison of PRELP between the AS-C and AS-N samples revealed that the amounts of PRELP were decreased in the AS-C areas compared to the AS-N areas ( $p=0.02$, Figure 2 (a) right).

We then investigated why the PRELP molecules showed so broad bands. Since PRELP is known to possess glycosylation, we removed $\mathrm{N}$-glycosylation of the protein samples by the treatment with PNGase, after which we similarly detected PRELP by1D-WB. As a result, the broad PRELP bands were not detected, instead, sharp bands with MW of $\sim 50 \mathrm{kDa}$ were detected (Figure 2 (b) left). This indicates that PRELP molecules possess various degrees of $\mathrm{N}$-glycosylation. To further confirm this, we detected PRELP molecules by 2D-WB. As shown in Figure 2(b) right, PRELP molecules were detected as at least 13 spots, in which the higher MW spots showed the lower $\mathrm{pI}$. When we detected PRELP molecules after removal of $\mathrm{N}$-glycosylation by PNGase, a single spot was detected instead of the multiple PRELP spots (Figure 2(b) right). Thus, the existence of the 13 PRELP spots indicates 13 types of PRELP molecules with different amounts of $\mathrm{N}$-glycosylation. This result confirmed that aortic valve tissue contains variously $\mathrm{N}$-glycosylated.

We then investigated whether high-glycosylated PRELP or lowglycosylated one was decreased. Specifically, in the result of 1D-WB, we divided the broad bands of PRELP into 3 parts with an identical size (high, moderate, and low MW-PRELP), as shown in Figure 2 (c) and then compared the ratios of the intensity of the 3 areas between the AS-C and AS-N areas. As a result, the amounts of high MW-PRELP did not show significant difference between the AS-C and AS-N areas, however, the amounts of moderate and low MW-PRELP were decreased in the AS-C areas compared to the AS-N areas as shown in Figure 2 (c). Similarly, we measured the intensity of the 13 PRELP spots in 2D-WB (Figure 2 (d)), 4 spots with relatively lower MWs and higher $\mathrm{pI}$ s showed decreased intensity in the AS-C areas compared to the AS-N areas (Figure 2(d)). These results are compatible with the results from $1 \mathrm{D}-\mathrm{WB}$.

Our results indicate that amounts of PRELP with moderate to low amounts of $\mathrm{N}$-glycosylation were decreased in the AS-C areas.

\section{Comparison of decorin between the AS-C and AS-N areas in AS aortic valves}

Second, we characterized decorin in the AS valves by $1 \mathrm{D}-\mathrm{WB}$. We detected two bands for decorin. The upper main bands showed approximate MWs of $90-130 \mathrm{kDa}$ and the lower bands showed $\sim 48$ $\mathrm{kDa}$ (Figure 3 (a) upper). As shown in Figure 3 (a) lower left, the total 
(a)
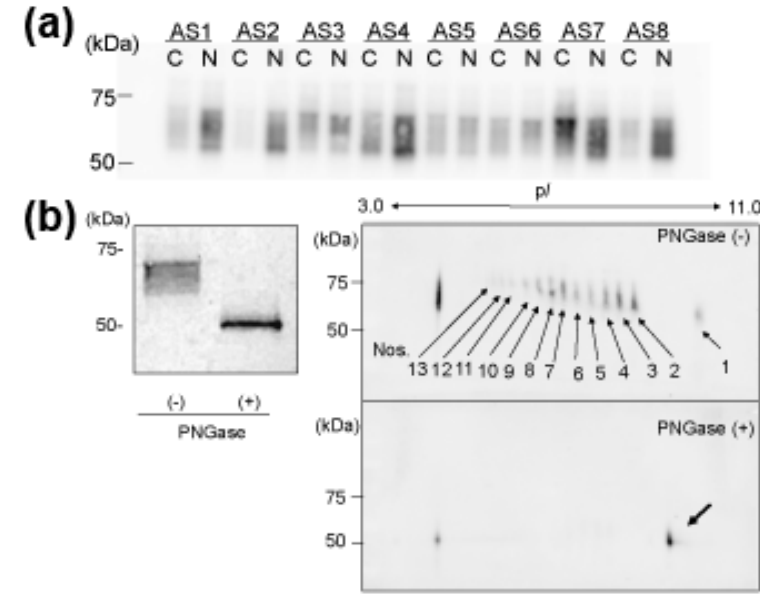

(C) $(\mathrm{kDa}) \frac{\mathrm{AS} 1}{\mathrm{CN}} \frac{\mathrm{AS} 2}{\mathrm{CN}} \frac{\mathrm{AS} 3}{\mathrm{CN}} \frac{\mathrm{AS} 4}{\mathrm{CN}} \frac{\mathrm{AS} 5}{\mathrm{CN}} \frac{\mathrm{AS6}}{\mathrm{C} N} \frac{\mathrm{AS7}}{\mathrm{C} N} \frac{\mathrm{AS} 8}{\mathrm{C} N}$

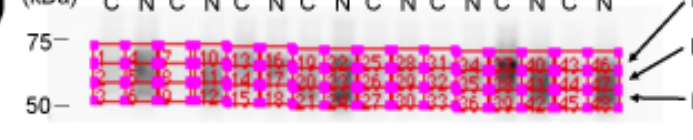

High MW-PRELP

(No8. $1,4,7,10,13,16,19,22,25,28,31,34,37,40,43,46)$ Moderate MW-PRELP

(Noe. $2,5,8,11,14,17,20,23,26,29,32,35,36,41,44,47)$ LOW MW-PRELP

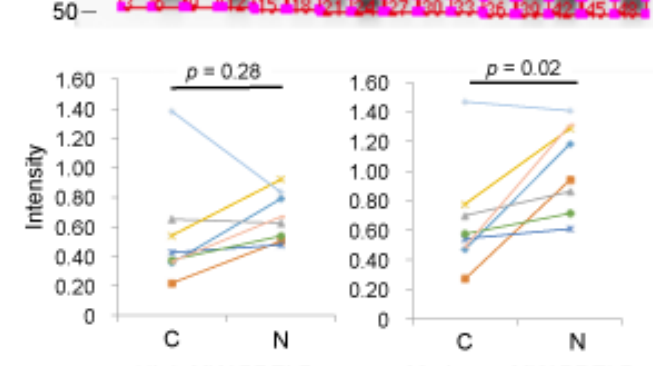

(Noe. $3,6,9,12,15,18,21,24,27,30,33,36,39,42,45,48$ )

(d)
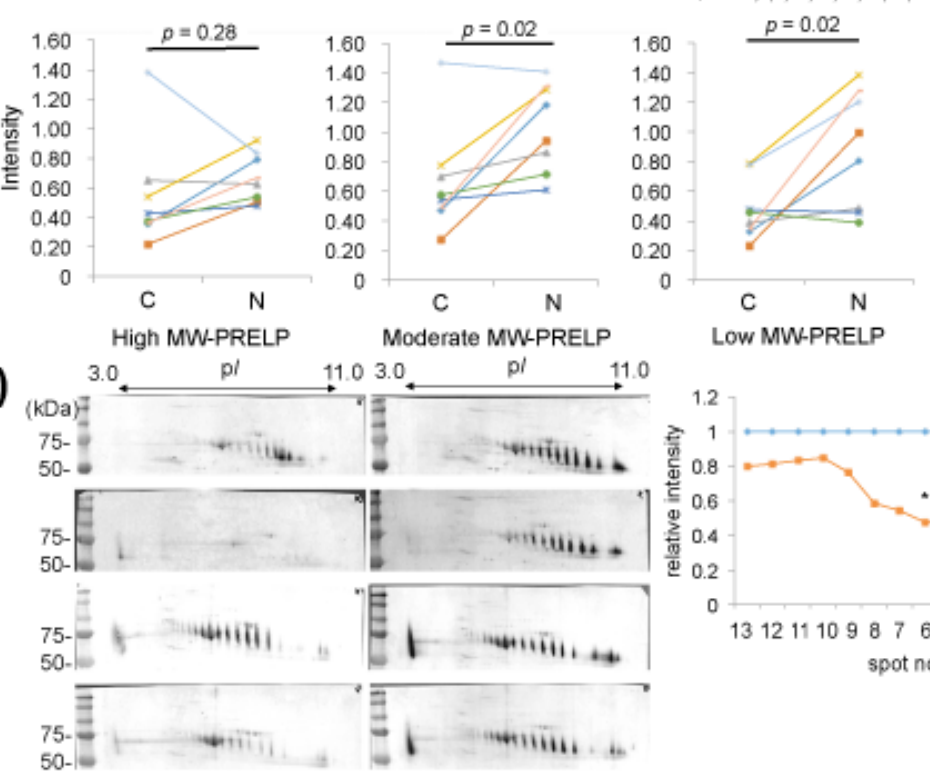

LOW MW-PRELP

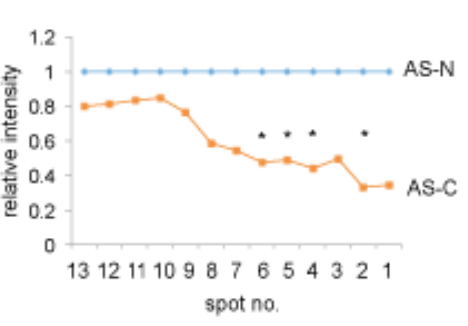

Figure 2. Analysis of PRELP in the AS-C and AS-N areas of AS aortic valves.

(a) Valve protein samples $(2.5 \mu \mathrm{g})$ were separated by $12.5 \%$ SDS-PAGE and PRELP was detected by WB using PVDF membranes, rabbit anti-human PRELP antibodies (x 1,000), HRPlabelled goat anti-rabbit IgG (x 5,000), and ECL Select system (left). The intensity the detected PRELP bands was compared between the AS-C and AS-N areas (right).

(b) Valve protein samples with or without the PNGase treatment were separated by $15 \%$ SDS-PAGE and PRELP was detected by WB using nitrocellulose membranes, rabbit anti-human PRELP antibodies (x 200), HRP-labelled goat anti-rabbit IgG (x 1,000), and 3, 3'-diaminobenzidine, tetrahydrochloride (DAB). A representative result from the AS-N sample of AS3 is shown (left). M; Marker. Similarly, valve protein samples with or without the PNGase treatment were separated by 2D gel electrophoresis and PRELP was detected by WB using PVDF membranes, rabbit anti-human PRELP antibodies (x 1,000), HRP-labelled goat anti-rabbit IgG (x 5,000), and ECL Select system (right). A representative result from the AS-C sample of AS8 is shown.

(c) The broad bands of PRELP shown in (a) were divided into 3 parts with an identical size (high, moderate, and low MW-PRELP). Then intensity of each of the 3 areas was compared the between the AS-C and AS-N areas.

(d) Seventy $\mu \mathrm{g}$ of samples with or without PNGase treatment were separated by $2 \mathrm{D}$ gel electrophoresis and then PRELP was detected by WB using nitrocellulose membranes rabbit antihuman PRELP antibodies (x 200), HRP-labelled goat anti-rabbit IgG (x 1,000), and DAB (left). The intensity of 13 PRELP spots was measured. The relative mean intensity of the spots in AS-C samples is shown. The mean intensity of the spots in AS-N samples is defined as 1.0 (right). Four samples (AS1, AS2, AS7, and AS8) amounts of which were enough for 2D-WB were analyzed. $* p<0.05$.

intensity of decorin was significantly lower in the AS-C areas than in the AS-N areas. This decrease was due to decrease of decorin with MWs of $90-130 \mathrm{kDa}$ (Figure 3 (a) lower middle), since the intensity of the decorin with MWs of $\sim 48 \mathrm{kDa}$ did not show significant difference between the 2 areas (Figure 3 (a) lower right).
Since decorin is known to possess $\mathrm{N}$ - or O-linked glycosylation, we characterized the $90-130 \mathrm{kDa}$ decorin and the $48 \mathrm{kDa}$ decorin from the aspect of glycosylation. We removed $\mathrm{N}$-glycosylation from the aortic valve proteins by PNGase treatment and then detected decorin by the same $1 \mathrm{D}-\mathrm{WB}$. As a result, the $48 \mathrm{kDa}$ decorin decreased its $\mathrm{MW}$ to 
approximately $40 \mathrm{kDa}$ (Figure $3(\mathrm{~b})$ ). Since the predicted $\mathrm{MW}$ of the mature decorin core protein is $36.3 \mathrm{kDa}$ [19-20], the $40 \mathrm{kDa}$ band was thought to be the core protein of decorin. The low MW-decorin was thought to be an N-glycosylated form of decorin. On the other hand, the high MW-decorin (90-130 kDa) also looked to decrease its MW by approximately $10 \mathrm{kDa}$ (Figure 3(b)). This decrease of MWs was also thought to be due to removal of $\mathrm{N}$-glycosylation. Thereby, the main modification of the high $\mathrm{MW}$-decorin $(90-130 \mathrm{kDa})$ is concluded to be not N-glycosylation but O-glycosylation. We concluded that 90-130 $\mathrm{kDa}$ decorin which possessed both $\mathrm{N}$-glycosylation and O-glycosylation was decreased in the AS-C area compared to the AS-N area.

\section{Comparison of biglycan between the AS-C and AS-N areas in AS aortic valves}

Finally, we compared biglycan in the AS valves. In $1 \mathrm{D}-\mathrm{WB}$, biglycan was detected as a broad band with MWs of $80-120 \mathrm{kDa}$ (high MW-biglycan) and that with $40 \mathrm{kDa}$ (low MW- biglycan), as shown in Figure 4(a)-upper left. The total amounts of biglycan showed no specific difference between the AS-C areas compared to the AS- $\mathrm{N}$ areas (Figure 4(a) upper). However, the ratio of high MW-biglycan to low MW-biglycan was decreased in the AS-C areas compared to the AS-N areas (Figure 4(a) lower middle and right). To investigate difference of the high and low MW-biglycan, we removed N-glycosylation from biglycan by the treatment with PNGase. As shown in Figure 4(b), the low MW-biglycan further decreased its MW from $40 \mathrm{kDa}$ down to approximately $35 \mathrm{kDa}$. This newly appeared $35 \mathrm{kDa}$ band was thought to be the core protein of biglycan, since the predicted MW of a mature biglycan core protein is $38.0 \mathrm{kDa}$ [20-21]. On the other hand, the high MW-biglycan decreased its MW from $80-120 \mathrm{kDa}$ down to approximately $70-110 \mathrm{kDa}$. Since biglycan is known to possess N- or O-linked glycosylation, our data indicate that both the high and low MW-biglycan molecules possess $\mathrm{N}$-glycosylation but only the high MW-biglycan possesses O-glycosylation. Collectively, the biglycan with $\mathrm{O}$-and $\mathrm{N}$-linked glycosylation was found decreased and biglycan with only $\mathrm{N}$-linked glycosylation was increased in the AS-C areas compared to the AS-N areas.

\section{Discussion}

We here characterized three SLRPs of PRELP, decorin, and biglycan in the AS valves. All the three SLRPs of the AS-C area showed quantitative and/or qualitative differences from those of the AS-N areas.

We here first showed that the amounts of PRELP with moderate and/or low amounts of $\mathrm{N}$-glycosylation were decreased in the AS-C areas. PRELP has been reported to possess various functions. Structurally, PRELP has been indicated to anchor basement membranes to connective tissue [22]. In the aspects of inflammation, PRELP has been reported to inhibit formation of complement membrane attack complex C5b-9 [23]. It has been reported that the complement system is activated in atherosclerotic lesions and the activation is deeply involved in the pathogenesis of atherosclerosis [24-27]. Activation of the complement system in AS valves has been also reported. The C5b-9 complex was found already in early AS valves and the deposition of the complex was augmented in advanced stenotic valves [28]. Moreover, $\mathrm{C} 3 \mathrm{aR}$ and C5aR was strongly expressed in the stenotic valves [28]. As above described, taking these reports together with our results, PRELP with decreased $\mathrm{N}$-glycosylation may promote complement activation by losing the inhibition of the C5b-9 complex formation. Further studies are needed to clarify this point.

Next, we here showed that $90-130 \mathrm{kDa}$ decorin which possessed both $\mathrm{N}$-glycosylation and O-glycosylation was decreased in the AS-C area compared to the AS-N area. Decorin possesses an N- or O-linked chondroitin sulfate (CS)/dermatan sulfate (DS)-chain in its N-terminal region and multiple $\mathrm{N}$-linked oligosaccharides [29]. Decorin was first thought to be a structural constituent of ECM, but was found to play roles in various aspects such as inflammation, calcification, and cell proliferation [30]. From the viewpoint of calcification, matrix metalloproteinase (MMP-9) is one of the key components of ECM degradation and bone mineralization [31,32]. MMP-9 has been reported (a)

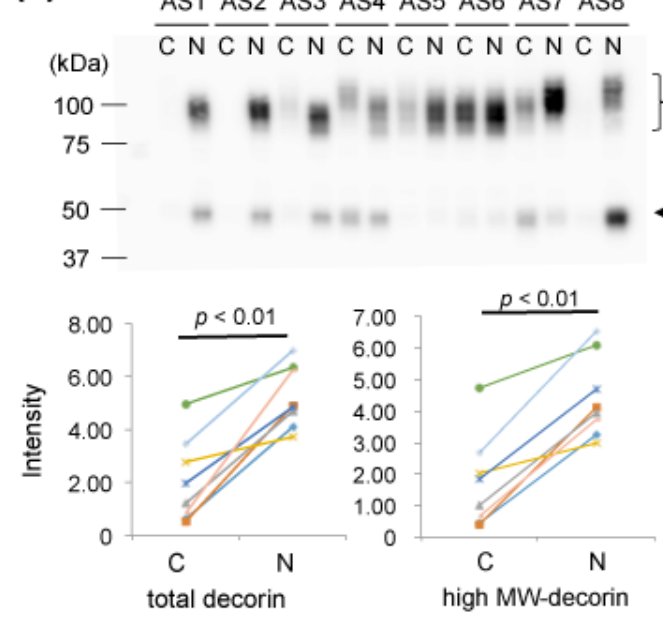

(b)

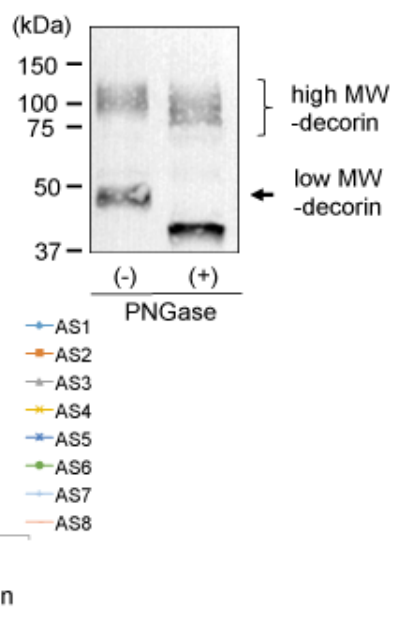

Figure 3. Comparison of decorin between the AS-C and -N areas.

(a) Valve protein samples $(2.5 \mu \mathrm{g})$ were separated by $12.5 \%$ SDS-PAGE and decorin was detected by WB using PVDF membranes, rabbit anti-human decorin antibodies (x 1,000), HRPlabelled goat anti-rabbit IgG (x 5,000), and ECL Select system (upper). The total intensity of both the approximately $90-130 \mathrm{kDa}$ bands and $50 \mathrm{kDa}$ bands was compared between the AS-C and AS-N areas (lower left). The intensity of the approximately $90-130 \mathrm{kDa}$ bands and $50 \mathrm{kDa}$ bands was separately compared between the AS-C and AS-N areas (lower middle and right, respectively).

(b) Valve protein samples with or without the PNGase treatment were separated by $15 \%$ SDS-PAGE and decorin was detected by WB using nitrocellulose membranes, rabbit anti-human decorin antibodies (x 100), HRP-labelled goat anti-rabbit $\operatorname{IgG}$ (x 2,000), and DAB. A representative result from the AS-C sample of AS8 is shown. 
(a)

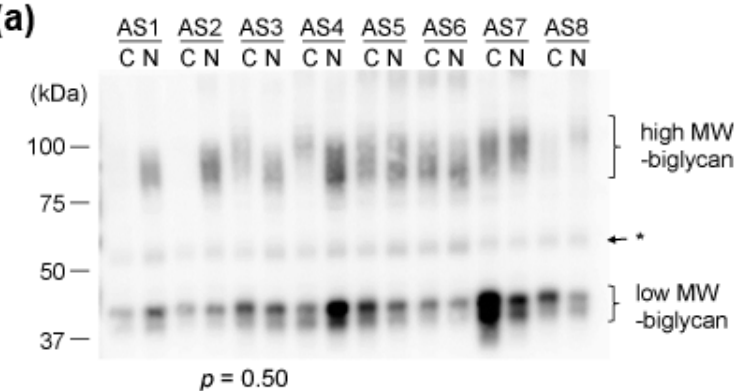

(b)

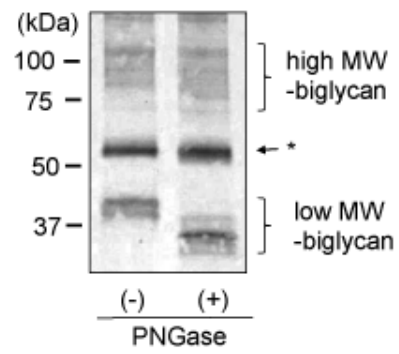

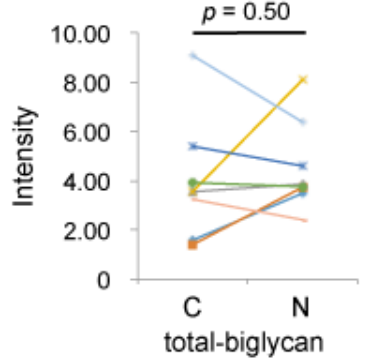
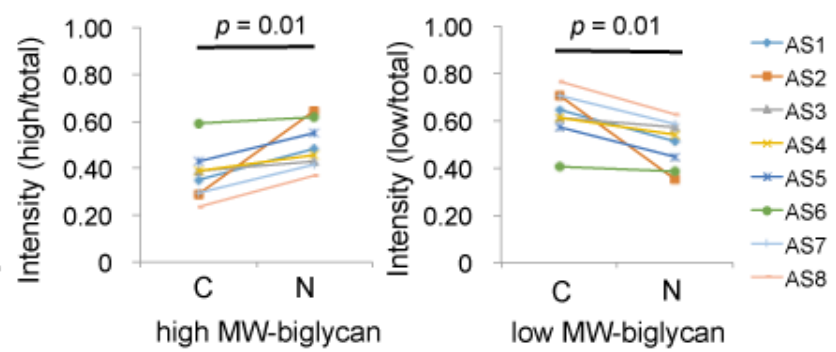

Figure 4. Comparison of biglycan between the AS-C and AS-N areas.

(a) Valve protein samples $(2.5 \mu \mathrm{g})$ were separated by $12.5 \%$ SDS-PAGE and biglycan was detected by WB using PVDF membranes, rabbit anti-human biglycan antibodies (x 1,000), HRP-labelled goat anti-rabbit IgG (x 2,000), and ECL Select system (upper). * The bands indicated by the arrow were not thought to be biglycan, since the bands were also detected in the negative control (data not shown).

(b) Valve protein samples $(5 \mu \mathrm{g})$ with or without the PNGase treatment were separated by $15 \%$ SDS-PAGE and biglycan was detected by WB using nitrocellulose membranes, rabbit antihuman biglycan antibodies (x 500), HRP-labelled goat anti-rabbit IgG (x 1,000), and DAB. A representative result from the AS-N sample of AS5 is shown.

to be increased in AS valves as well as abdominal aortic aneurism $[33,34]$. Moreover, MMP-9 is thought to be involved in extraosseous mineralization $[35,36]$. Considering these reports, MMP-9 is expected to be involved in the calcification process of AS valves. Recently, decorin was reported to inhibit secretion of MMP-9 in vascular smooth muscle cells [33]. Thereby, one of the physiological roles of decorin may be protection of aortic valves from ECM degradation and mineralization by inhibiting MMP-9. The decrease of highly glycosylated decorin detected here may lose the protective function, leading to progression of mineralization in AS valves.

Finally, we found the aberrant glycosylation of biglycan. Biglycan consists of a $42 \mathrm{kDa}$ core protein, two GAG chains consisting of DS or CS, and N-linked oligosaccharides [37]. Biglycan interacts with collagen fibrils both via the core protein and GAG chains [38]. Biglycan without GAG increases with age in human articular cartilage [39]. Biglycan is suggested to play a role in the mineralization of bone $[40,41]$. Moreover, biglycan is thought to promote innate immunity and increase proinflammatory cytokine production [42]. In our study, the ratios of biglycan with GAG to that without GAG were decreased in the AS-C areas. Although roles of glycosylation in mineralization and inflammation are still unclear, the biglycan with decreased glycosylation might promote inflammation and mineralization in AS valves. Further studies are needed to clarify this point.

\section{Conclusions}

We here reported that quantity and glycosylation condition of PRELP, decorin, and biglycan were altered in the calcified and thickened area of AS valves. These alternations may promote AS changes of aortic valves from the aspects of inflammation and mineralization.

\section{Conflict of interest statement}

None.

\section{References}

1. Newby DE, Cowell SJ, Boon NA. (2006) Emerging medical treatments for aortic stenosis: statins, angiotensin converting enzyme inhibitors, or both? Heart 92: 729734. [Crossref]

2. RabuÅŸ MB, Kayalar N, Sareyy $\tilde{A} 1 / 4$ po ÄŸlu B, Erkin A, Kirali K, et al. (2009) Hypercholesterolemia association with aortic stenosis of various etiologies. $J$ Card Surg 24: 146-150. [Crossref]

3. O'Brien KD, Reichenbach DD, Marcovina SM, Kuusisto J, Alpers CE, et al. (1996) Apolipoproteins B, (a), and E accumulate in the morphologically early lesion of 'degenerative' valvular aortic stenosis. Arterioscler Thromb Vasc Biol 16: 523-532. [Crossref]

4. Otto CM, Kuusisto J, Reichenbach DD, Gown AM, O'Brien KD (1994) Characterization of the early lesion of 'degenerative' valvular aortic stenosis. Histological and immunohistochemical studies. Circulation 90: 844-853. [Crossref]

5. Mohler ER 3rd, Gannon F, Reynolds C, Zimmerman R, Keane MG, et al. (2001) Bone formation and inflammation in cardiac valves. Circulation 103: 1522-1528. [Crossref]

6. Miller JD, Weiss RM, Heistad DD (2011) Calcific aortic valve stenosis: methods models, and mechanisms. Circ Res 108: 1392-1412. [Crossref]

7. Winchester R, Wiesendanger M, O'Brien W, Zhang HZ, Maurer MS, et al. (2011) Circulating activated and effector memory $\mathrm{T}$ cells are associated with calcification and clonal expansions in bicuspid and tricuspid valves of calcific aortic stenosis. $J$ Immunol 187: 1006-1014. [Crossref]

8. Taylor PM1 (2007) Biological matrices and bionanotechnology. Philos Trans R Soc Lond B Biol Sci 362: 1313-1320. [Crossref]

9. Culav EM, Clark CH, Merrilees MJ (1999) Connective tissues: matrix composition and its relevance to physical therapy. Phys Ther 79: 308-319. [Crossref]

10. Suzuki H, Chikada M, Yokoyama MK, Kurokawa MS, Ando T, et al. (2016) Aberrant Glycosylation of Lumican in Aortic Valve Stenosis Revealed by Proteomic Analysis. Int Heart J 57: 104-111. [Crossref]

11. Roughley PJ1 (2006) The structure and function of cartilage proteoglycans. Eur Cell Mater 12: 92-101. [Crossref]

12. McEwan PA, Scott PG, Bishop PN, Bella J (2006) Structural correlations in the family of small leucine-rich repeat proteins and proteoglycans. J Struct Biol 155: 294-305. [Crossref]

13. Kalamajski S, Oldberg A (2010) The role of small leucine-rich proteoglycans in 
collagen fibrillogenesis. Matrix Biol 29: 248-253. [Crossref]

14. Vogel KG, Paulsson M, Heinegård D (1984) Specific inhibition of type I and type II collagen fibrillogenesis by the small proteoglycan of tendon. Biochem J 223: 587-597. [Crossref]

15. Funderburgh JL, Mitschler RR, Funderburgh ML, Roth MR, Chapes SK, et al. (1997) Macrophage receptors for lumican. A corneal keratan sulfate proteoglycan. Invest Ophthalmol Vis Sci 38: 1159-1167. [Crossref]

16. Hayashi Y, Call MK, Chikama T, Liu H, Carlson EC, et al. (2010) Lumican is required for neutrophil extravasation following corneal injury and wound healing. $J$ Cell Sci 123: 2987-2995. [Crossref]

17. Carlson EC, Sun Y, Auletta J, Kao WW, Liu CY, et al. (2010) Regulation of corneal inflammation by neutrophil-dependent cleavage of keratan sulfate proteoglycans as a model for breakdown of the chemokine gradient. J Leukoc Biol 88: 517-522. [Crossref]

18. Matsuo K, Xiang Y, Nakamura H, Masuko K, Yudoh K, et al. (2006) Identification of novel citrullinated autoantigens of synovium in rheumatoid arthritis using a proteomic approach. Arthritis Res Ther 8: R175. [Crossref]

19. Krusius T, Ruoslahti E (1986) Primary structure of an extracellular matrix proteoglycan core protein deduced from cloned cDNA. Proc Natl Acad Sci U S A 83: 7683-7687. [Crossref]

20. Roughley PJ, White RJ (1989) Dermatan sulphate proteoglycans of human articular cartilage. The properties of dermatan sulphate proteoglycans I and II. Biochem J 262: 823-827. [Crossref]

21. Fisher LW, Termine JD, Young MF (1989) Deduced protein sequence of bone small proteoglycan I (biglycan) shows homology with proteoglycan II (decorin) and several nonconnective tissue proteins in a variety of species. J Biol Chem 264: 4571-4576. [Crossref]

22. Bengtsson E, Neame PJ, Heinegård D, Sommarin Y (1995) The primary structure of a basic leucine-rich repeat protein, PRELP, found in connective tissues. J Biol Chem 270 : 25639-25644. [Crossref]

23. Happonen KE, Fürst CM, Saxne T, Heinegård D, Blom AM (2012) PRELP protein inhibits the formation of the complement membrane attack complex. J Biol Chem 287: 8092-8100. [Crossref]

24. Niculescu F, Soane L, Badea T, Shin M, Rus H (1999) Tyrosine phosphorylation and activation of Janus kinase 1 and STAT3 by sublytic C5b-9 complement complex in aortic endothelial cells. Immunopharmacology 42: 187-193. [Crossref]

25. Vlaicu R, Niculescu F, Rus HG, Cristea A. (1985) Immunohistochemical localization of the terminal C5b-9 complement complex in human aortic fibrous plaque. Atherosclerosis 57(2-3): 163-177. [Crossref]

26. Laine P, Pentikäinen MO, Würzner R, Penttilä A, Paavonen T, et al. (2002) Evidence for complement activation in ruptured coronary plaques in acute myocardial infarction. $\mathrm{Am}$ J Cardiol 90: 404-408. [Crossref]

27. Oksjoki R, Kovanen PT, Pentikäinen MO (2003) Role of complement activation in atherosclerosis. Curr Opin Lipidol 14: 477-482. [Crossref]

28. Helske S, Oksjoki R, Lindstedt KA, Lommi J, Turto H, et al. (2008) Complement system is activated in stenotic aortic valves. Atherosclerosis 196: 190-200. [Crossref]

29. Sjöberg AP, Manderson GA, Mörgelin M, Day AJ, Heinegård D, et al. (2009) Short leucine-rich glycoproteins of the extracellular matrix display diverse patterns of complement interaction and activation. Mol Immunol 46: 830-839. [Crossref]

30. Järvinen TA, Prince S2 (2015) Decorin: A Growth Factor Antagonist for Tumor Growth Inhibition. Biomed Res Int 2015: 654765. [Crossref]

31. Nyman JS, Lynch CC, Perrien DS, Thiolloy S, O'Quinn EC, et al. (2011) Differentia effects between the loss of MMP-2 and MMP-9 on structural and tissue-level properties of bone. J Bone Miner Res 26: 1252-1260. [Crossref]

32. Rybakowski JK (2009) Matrix Metalloproteinase-9 (MMP9)-A Mediating Enzyme in Cardiovascular Disease, Cancer, and Neuropsychiatric Disorders. Cardiovasc Psychiatry Neurol.

33. Ueda K, Yoshimura K2, Yamashita O, Harada T, Morikage N, et al. (2015) Possible dual role of decorin in abdominal aortic aneurysm. PLoS One 10: e0120689. [Crossref]

34. Hellenthal FA, Buurman WA, Wodzig WK, Schurink GW (2009) Biomarkers of AAA progression. Part 1: extracellular matrix degeneration. Nat Rev Cardiol 6: 464-474. [Crossref]

35. Vyavahare N, Jones PL, Tallapragada S, Levy RJ (2000) Inhibition of matrix metalloproteinase activity attenuates tenascin-C production and calcification of implanted purified elastin in rats. Am J Pathol 157: 885-893. [Crossref]

36. Chen NX, O'Neill KD, Chen X, Kiattisunthorn K, Gattone VH, et al. (2011) Activation of arterial matrix metalloproteinases leads to vascular calcification in chronic kidney disease. Am J Nephrol 34: 211-219. [Crossref]

37. Nastase MV, Young MF, Schaefer L (2012) Biglycan: a multivalent proteoglycan providing structure and signals. J Histochem Cytochem 60: 963-975. [Crossref]

38. Schönherr E, Witsch-Prehm P, Harrach B, Robenek H, Rauterberg J, et al. (1995) Interaction of biglycan with type I collagen. $J$ Biol Chem 270: 2776-2783. [Crossref]

39. Roughley PJ, White RJ, Magny MC, Liu J, Pearce RH, et al. (1993) Non-proteoglycan forms of biglycan increase with age in human articular cartilage. Biochem J 295: 421426. [Crossref]

40. Xu T, Bianco P, Fisher LW, Longenecker G, Smith E, et al. (1998) Targeted disruption of the biglycan gene leads to an osteoporosis-like phenotype in mice. Nat Genet 20: 78-82. [Crossref]

41. Parisuthiman D, Mochida Y, Duarte WR, Yamauchi M (2005) Biglycan modulates osteoblast differentiation and matrix mineralization. J Bone Miner Res 20: 1878-1886. [Crossref]

42. Schaefer L, Babelova A, Kiss E, Hausser HJ, Baliova M, et al. (2005) The matrix component biglycan is proinflammatory and signals through Toll-like receptors 4 and 2 in macrophages. J Clin Invest 115: 2223-2233. [Crossref]

Copyright: (C2016 Furukawa H. This is an open-access article distributed under the terms of the Creative Commons Attribution License, which permits unrestricted use, distribution, and reproduction in any medium, provided the original author and source are credited. 